\title{
A High-Throughput Enzyme-Coupled Activity Assay to Probe Small Molecule Interaction with the dNTPase SAMHD1
}

\author{
Miriam Yagüe-Capilla ${ }^{1}$, Sean G. Rudd ${ }^{1}$ \\ ${ }^{1}$ Science for Life Laboratory, Department of Oncology-Pathology, Karolinska Institutet
}

\section{Corresponding Author}

Sean G. Rudd

sean.rudd@scilifelab.se

\section{Citation}

Yagüe-Capilla, M., Rudd, S.G. A

High-Throughput Enzyme-Coupled

Activity Assay to Probe Small

Molecule Interaction with the dNTPase

SAMHD1. J. Vis. Exp. (170), e62503,

doi:10.3791/62503 (2021).

\section{Date Published}

April 16, 2021

DOI

$10.3791 / 62503$

URL

jove.com/video/62503

\section{Abstract}

Sterile alpha motif and HD domain-containing protein 1 (SAMHD1) is a pivotal regulator of intracellular deoxynucleoside triphosphate (dNTP) pools, as this enzyme can hydrolyze dNTPs into their corresponding nucleosides and inorganic triphosphates. Due to its critical role in nucleotide metabolism, its association to several pathologies, and its role in therapy resistance, intense research is currently being carried out for a better understanding of both the regulation and cellular function of this enzyme. For this reason, development of simple and inexpensive highthroughput amenable methods to probe small molecule interaction with SAMHD1, such as allosteric regulators, substrates, or inhibitors, is vital. To this purpose, the enzyme-coupled malachite green assay is a simple and robust colorimetric assay that can be deployed in a 384-microwell plate format allowing the indirect measurement of SAMHD1 activity. As SAMHD1 releases the triphosphate group from nucleotide substrates, we can couple a pyrophosphatase activity to this reaction, thereby producing inorganic phosphate, which can be quantified by the malachite green reagent through the formation of a phosphomolybdate malachite green complex. Here, we show the application of this methodology to characterize known inhibitors of SAMHD1 and to decipher the mechanisms involved in SAMHD1 catalysis of noncanonical substrates and regulation by allosteric activators, exemplified by nucleosidebased anticancer drugs. Thus, the enzyme-coupled malachite green assay is a powerful tool to study SAMHD1, and furthermore, could also be utilized in the study of several enzymes which release phosphate species.

\section{Introduction}

Sterile alpha motif and histidine-aspartate domain-containing protein 1 (SAMHD1) is a central regulator of nucleotide homeostasis in mammalian cells ${ }^{1}$ with many roles in human health and disease ${ }^{2}$. This enzyme is capable of 
hydrolyzing deoxynucleoside triphosphates (dNTPs) into their cognate deoxynucleoside and inorganic triphosphate molecules $^{3,4}$, with this activity being allosterically regulated by (d)NTP abundance (reviewed in reference ${ }^{5}$ ). Each SAMHD1 monomer contains two allosteric sites (AS1 and AS2) and one catalytic site, and the formation of the active enzyme requires the ordered assembly of a homotetramer upon (d)NTP binding. Dimerization of SAMHD1 monomers is first triggered through the binding of a guanine triphosphate (GTP or dGTP) to AS1, and subsequent tetramerization is achieved when an additional dNTP molecule binds to AS2, enabling substrate access to the catalytic site and subsequent hydrolysis.

SAMHD1 substrates include the four canonical dNTPs ${ }^{3,4}$ together with some base and sugar modified nucleotides, including the triphosphate metabolites of several nucleosidebased drugs used in the treatment of viral infections and cancer, several of which can also serve as allosteric activators $6,7,8,9,10,11$. In consequence SAMHD1 modulates the efficacy of many of these compounds in disease models $7,8,9,10,11,12,13,14,15$, and furthermore, in the case of the deoxycytidine analogue cytarabine (araC), which has remained standard-of-care therapy for acute myeloid leukemia (AML) for decades, actually dictates treatment efficacy in this disease ${ }^{7,8,16}$. SAMHD1 is thus a potential biomarker and therapeutic target to improve the efficacy of nucleoside-based therapies ${ }^{17}$, and accordingly, we and others have sought to identify strategies to inactivate SAMHD1 in cells. We proposed the use of viral protein $X(V p x)$ as a biological inhibitor to target SAMHD1 for degradation inside cancer cells ${ }^{7}$, however, this approach has a number of limitations (discussed in reference ${ }^{12}$ ), and we also recently reported an indirect approach to suppress SAMHD1 activity via inhibition of ribonucleotide reductase which we demonstrated in various models of $\mathrm{AML}^{18}$. A number of studies have sought to identify small molecules capable of directly inhibiting SAMHD1, and to date, several such molecules have been reported, however, only documenting inhibition in vitro $6,9,19,20,21,22$. In consequence, a lack of small molecules that potently inhibit SAMHD1 activity in cells coupled with the complex mechanisms of SAMHD1 catalysis of nucleoside-based therapeutics, underscores the need for further investigation. Thus robust and ideally high-throughput amenable methods for probing small molecule interaction with SAMHD1 are ideal in order to identify substrates, allosteric regulators and inhibitors, of this clinically relevant enzyme.

Several methodologies are available that directly measure the dNTPase activity of SAMHD1, such as thin-layer chromatography (TLC) $)^{9,20,23}$ and high-performance liquid chromatography (HPLC) $)^{9,21}$, but these are not readily amenable to high-throughput setups. One exception is the assay reported by Mauney et al., which exploits the ability of SAMHD1 to hydrolyze bis (4-nitrophenyl) phosphate (b4NPP) to $\mathrm{p}$-nitrophenol and p-nitrophenyl phosphate when $\mathrm{Mn}^{2+}$ is used as the activating cation, resulting in a colorimetric change that can be readily measured in a microwell plate ${ }^{21}$. This assay has been successfully used for the identification and characterization of SAMHD1 inhibitors, but it should be noted that hydrolysis does occur in the absence of (d)NTP activators and in the presence of a likely non-physiological activating cation, both being important caveats to consider. This also renders this assay less applicable to the study and identification of allosteric regulators of SAMHD1.

In this context, an enzyme-coupled approach combined with the malachite green reagent, as detailed in this report, can be a versatile method to indirectly measure the dNTPase 
activity of SAMHD1 and, furthermore, interrogate the impact of various small molecules upon it. The malachite green assay is a robust and reliable colorimetric technique for the detection of free inorganic phosphate $(\mathrm{Pi})$, based on the formation of a molybdophosphoric acid complex that leads to a colorimetric change measured at $620 \mathrm{~nm}^{24}$. As SAMHD1 hydrolysis releases the triphosphate group from nucleotide substrates, it is thus necessary to couple this reaction with a (pyro)phosphatase activity, which will generate free inorganic phosphate, prior to the addition of the malachite green reagent. The malachite green assay is sensitive and cost effective and has been widely used for the identification and characterization of inhibitors and substrates for enzymes that release inorganic phosphate groups either in their reactions or in the presence of a coupling enzyme. It has been widely applied in characterization of the ATPase activities of helicases $25,26,27$, or the study of CD73 enzymatic activity, which mediates the degradation of AMP to adenosine and inorganic phosphate ${ }^{28}$. Additionally, when coupled, it has been employed in the discovery of antibiotic drugs targeting the UDP-2,3-diacylglucosamine pyrophosphatase $\mathrm{LpxH}$, an essential enzyme in most Gram-negative pathogens ${ }^{29}$. With regards to cancer research, the enzyme-coupled approach has been extensively deployed against the NUDIX hydrolases, a family of nucleotide metabolizing enzymes, both in the characterization of substrates ${ }^{30,31,32}$ and in the identification and development of drugs and chemical

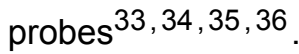

With regards to the dNTPase SAMHD1, this approach has been utilized in several reports. Using exopolyphosphatase Ppx1 from Saccharomyces cerevisiae as the coupling enzyme, this assay was used to test several nucleotide analogues as either substrates, activators, or inhibitors of SAMHD1, and resulted in the identification of the triphosphate metabolite of the anti-leukemic drug clofarabine as an activator and substrate ${ }^{6}$. Additionally, with inorganic pyrophosphatase from Escherichia coli as the coupling enzyme, it has been employed in the screening of a library of clinically approved compounds against SAMHD1 to identify inhibitors ${ }^{20}$. In our research, we utilized this approach to show that ara-CTP, the active metabolite of ara-C, is a SAMHD1 substrate but not allosteric activator ${ }^{7}$ and subsequently used this assay to show that several small molecules that could sensitize AML models to ara-C in a SAMHD1-dependent manner, actually did not directly inhibit SAMHD ${ }^{18}$. In this report, we will detail this versatile method and demonstrate its applicability, in a high-throughput amenable setup, for the identification of inhibitors, activators, and substrates of SAMHD1.

\section{Protocol}

A schematic overview of the methods below is depicted in Figure 1 and a detailed list of materials and reagents is available in the Table of Materials.

\section{Preparation of assay buffers.}

\section{Preparation of stock buffers.}

NOTE: As the assay is sensitive to the detection of phosphates, which can be commonplace, rinse glassware three times with ultrapure or double-distilled water to avoid contamination. All buffers can be stored at room temperature (RT).

1. Prepare $1 \mathrm{~L}$ of $\mathrm{SAMHD} 1$ reaction buffer $(\mathrm{RB})$ stock solution (25 mM Tris-Acetate $\mathrm{pH} 8,40 \mathrm{mM} \mathrm{NaCl}, 1$ $\mathrm{mM} \mathrm{MgCl} 2$ ) by dissolving $4.5 \mathrm{~g}$ Tris Acetate, $2.3 \mathrm{~g}$ $\mathrm{NaCl}$, and $0.2 \mathrm{~g} \mathrm{MgCl}_{2}$, in approximately $800 \mathrm{~mL}$ of water before adjusting to $\mathrm{pH} 8$ and final volume. 
2. Prepare $5 \mathrm{~mL}$ of $0.1 \mathrm{M}$ TCEP stock solution by diluting $1 \mathrm{~mL}$ of $0.5 \mathrm{M}$ TCEP into $4 \mathrm{~mL}$ of water.

3. Prepare $50 \mathrm{~mL}$ of $11 \%$ Tween-20 stock solution by diluting $5 \mathrm{~mL}$ of $100 \%$ Tween-20 into $44.5 \mathrm{~mL}$ of water.

NOTE: Tween-20 is light sensitive.

4. Prepare $50 \mathrm{~mL}$ of $0.5 \mathrm{M}$ EDTA stop solution by dissolving $9.3 \mathrm{~g}$ EDTA in approximately $40 \mathrm{~mL}$ of water before adjusting to $\mathrm{pH} 8$ and final volume.

5. Prepare Malachite Green (MG) stock solution (3.2 $\mathrm{mM}$ malachite green in $\mathrm{H}_{2} \mathrm{SO}_{4}$ ) by slowly adding 60 $\mathrm{mL}$ concentrated sulfuric acid to $300 \mathrm{~mL}$ water in a brown glass bottle. Cool the solution to RT and dissolve $0.44 \mathrm{~g}$ malachite green.

CAUTION: The reaction of sulfuric acid with water is exothermic and so the bottle may heat up causing a build-up of pressure; ensure this pressure is released frequently.

NOTE: The resulting orange solution is light sensitive (hence brown bottle) and stable for at least 1 year at RT. Precipitate may form over time, ensure only the supernatant is used.

6. Prepare $50 \mathrm{~mL}$ of $7 \%$ ammonium molybdate stock solution by dissolving $3.75 \mathrm{~g}$ ammonium molybdate in $50 \mathrm{~mL}$ of water.

NOTE: Precipitate may form over time, ensure only the supernatant is used.

\section{Preparation of complete assay buffers}

NOTE: This should be done on the day of the experiment

1. Prepare complete SAMHD1 RB (25 mM TrisAcetate $\mathrm{pH} 8,40 \mathrm{mM} \mathrm{NaCl}, 1 \mathrm{mM} \mathrm{MgCl} 2,0.3 \mathrm{mM}$ TCEP, $0.005 \%$ Tween-20). Use previously prepared $11 \%$ Tween-20 and $0.1 \mathrm{M}$ TCEP stocks to add these components at a final concentration of $0.005 \%$ for Tween-20 and $0.3 \mathrm{mM}$ for TCEP to the SAMHD1 RB stock.

2. Prepare EDTA stop solution (25 mM Tris-Acetate $\mathrm{pH}$ $8,40 \mathrm{mM} \mathrm{NaCl}, 1 \mathrm{mM} \mathrm{MgCl} 2,0.3 \mathrm{mM}$ TCEP, $0.005 \%$ Tween-20, 7.9 mM EDTA). To complete SAMHD1 $\mathrm{RB}$, use $0.5 \mathrm{M}$ EDTA stock solution to add EDTA to a final concentration of $7.9 \mathrm{mM}$.

3. Prepare MG working solution (2.5 mM malachite green, $1.4 \%$ ammonium molybdate, $0.18 \%$ Tween-20) by mixing 10 parts of MG stock solution with 2.5 parts of $7 \%$ ammonium molybdate and 0.2 parts of $11 \%$ Tween-20.

\section{SAMHD1 inhibition assay and determination of compound IC 50}

NOTE: Final assay conditions are shown in Table 1.

\section{Preparation of compounds in assay plate}

NOTE: Small molecular weight compounds are typically dissolved in 100\% DMSO and nucleotide analogues in water. Stock concentration ranges from 10 to $100 \mathrm{mM}$ and is influenced by the potency and solubility of the compounds, together with the DMSO tolerance of the assay. Check that the final DMSO concentration in the reaction does not exceed $1 \%$ to ensure enzyme activities are not affected by this solvent. It is good practice to test the tolerance of the assay to the solvent prior to the experiment.

1. Prepare serially diluted test compounds at $100 x$ final concentration in the relevant solvent (e.g., 100\% DMSO for small molecules or water for nucleotide analogues) in a clear round-bottomed polypropylene 
96-well plate using either a multichannel pipette or automated liquid handling equipment.

NOTE: Depending upon compound stability, dilution plates can be prepared in advance, sealed, and stored at $-20{ }^{\circ} \mathrm{C}$. Allow plates to equilibrate to RT before continuing the protocol.

2. Using complete SAMHD1 RB, dilute compounds to $25 x$ final concentration (to maintain the final solvent concentration below 1\%) and transfer $5 \mu \mathrm{L}$ to the appropriate wells of a clear 384-well flat-bottomed assay plate. Repeat the procedure with solvent-only control samples.

\section{Preparation of reaction components}

NOTE: This should be done on the day of the assay. Recombinant human SAMHD1 and E. coli pyrophosphatase (PPase) aliquots are stored long term at $-80{ }^{\circ} \mathrm{C}$ diluted at $9.1 \mathrm{mg} / \mathrm{mL}$ and $23.0 \mathrm{mg} / \mathrm{mL}$, respectively, in storage buffer (20 mM HEPES pH 7.5, $300 \mathrm{mM} \mathrm{NaCl}, 10 \%$ glycerol, $2 \mathrm{mM}$ TCEP). Once thawed, aliquots are stored short-term at $-20^{\circ} \mathrm{C}$.

1. Prepare enzyme (SAMHD1/PPase) master mix by diluting recombinant human SAMHD1 protein and recombinant PPase in complete SAMHD1 RB to $4 x$ desired final concentration, thus $1.4 \mu \mathrm{M}$ SAMHD1 and $50 \mathrm{U} / \mathrm{mL}$ PPase.

2. Prepare activator/substrate dGTP by diluting dGTP stock (typically 10 or $100 \mathrm{mM}$ in water) in complete SAMHD1 RB to $2 x$ final concentration, thus $50 \mu \mathrm{M}$ dGTP.

\section{Perform the assay}

NOTE: All assay components should be equilibrated to RT. Liquid additions can be performed with either a multichannel pipette or a bulk reagent liquid dispenser.
1. To 384-well assay plate containing compound dilutions and solvent only controls, dispense $5 \mu \mathrm{L}$ of SAMHD1/PPase master mix. To no enzyme control wells, dispense $5 \mu \mathrm{L}$ of complete SAMHD1 RB. Preincubate enzyme and compounds for $10 \mathrm{~min}$ at RT.

2. To all wells, dispense $10 \mu \mathrm{L}$ of $2 \mathrm{x}$ dGTP solution to start the reaction.

3. Incubate the reaction for $20 \mathrm{~min}$ at RT.

4. Stop the reaction by dispensing $20 \mu \mathrm{L}$ EDTA stop solution to all wells.

NOTE: The experiment can be paused here if desired.

5. Add $10 \mu \mathrm{L} M G$ working solution to all wells.

CAUTION: MG working solution contains sulfuric acid.

6. Ensure mixing of well contents using an orbital microwell plate shaker and centrifugation at 1,000 $\mathrm{x}$ $g$ for $1 \mathrm{~min}$.

7. Incubate the plate for $20 \mathrm{~min}$ at RT.

8. Read the absorption at $630 \mathrm{~nm}$ wavelength in a microwell plate reader.

\section{Data visualization and analysis}

1. Calculate the average and standard deviation of the positive and negative control wells (positive, complete reaction with solvent; negative, dGTP alone with solvent). Calculate Z-factor ${ }^{37}$ as an indicator of assay quality.

2. Normalize each absorbance value to the mean values of the positive and negative controls, setting the positive control as 100\% SAMHD1 activity and the negative control as 0\% SAMHD1 activity. 
3. Plot SAMHD1 activity (\%) as a function of compound concentration and fit a four-parameter variable slope dose-response curve, allowing determination of compound $\mathrm{IC}_{50}$.

\section{SAMHD1 activator and substrate screen}

NOTE: Final assay conditions are shown in Table 2. Recombinant SAMHD1 and PPase aliquots are stored long term at $-80{ }^{\circ} \mathrm{C}$ diluted at $9.1 \mathrm{mg} / \mathrm{mL}$ and $23.0 \mathrm{mg} / \mathrm{mL}$, respectively, in storage buffer $(20 \mathrm{mM}$ HEPES pH 7.5, 300 $\mathrm{mM} \mathrm{NaCl}, 10 \%$ glycerol, $2 \mathrm{mM}$ TCEP) at $-80^{\circ} \mathrm{C}$. Once thawed, aliquots are stored short term at $-20^{\circ} \mathrm{C}$.

\section{Preparation of nucleotide analogues in assay plate}

1. Dilute nucleotide analogue stocks (typically 10 or $100 \mathrm{mM}$ in water) to $4 \mathrm{x}$ final concentration in complete SAMHD1 RB, in our case $800 \mu \mathrm{M}$ nucleotide analogue, and transfer $5 \mu \mathrm{L}$ to the appropriate wells of a 384-well assay plate.

\section{Preparation of reaction components}

NOTE: This should be done on the day of the assay

1. Prepare enzyme (SAMHD1/PPase) master mix by diluting recombinant human SAMHD1 protein and recombinant E. coli PPase in complete SAMHD1 RB to $2 x$ desired final concentration, thus $0.7 \mu \mathrm{M}$ SAMHD1 and $25 \mathrm{U} / \mathrm{mL}$ PPase.

2. Prepare PPase alone solution by diluting recombinant E. coli PPase in complete SAMHD1 RB to $2 x$ desired final concentration, thus $25 \mathrm{U} / \mathrm{mL}$ PPase.

3. Prepare activators GTP (AS1) and dGTPaS (AS1 and AS2) diluting stock (typically 10 or $100 \mathrm{mM}$ in water) in complete SAMHD1 RB to $4 \mathrm{x}$ final concentration, thus $50 \mu \mathrm{M}$ GTP or dGTPaS.

\section{Perform the assay}

NOTE: All assay components should be equilibrated to RT. Liquid additions can be performed with either a multichannel pipette or a bulk reagent liquid dispenser.

1. To 384-well assay plate containing nucleotide analogues, dispense $5 \mu \mathrm{L}$ of the activator (either GTP or dGTPaS) or complete SAMHD1 RB to the appropriate wells.

2. Start the reaction by dispensing $10 \mu \mathrm{L}$ of SAMHD1/ PPase master mix, PPase alone, or complete SAMHD1 RB to the appropriate wells.

3. Incubate the reaction for $20 \mathrm{~min}$ at RT.

4. Stop the reaction by dispensing $20 \mu \mathrm{L}$ EDTA stop solution to all the wells.

NOTE: The experiment can be paused here if desired.

5. Add $10 \mu \mathrm{L} M G$ working solution to all the wells.

CAUTION: MG working solution contains sulfuric acid.

6. Ensure mixing of well contents using an orbital microwell plate shaker and centrifugation at $1,000 \mathrm{x}$ $g$ for $1 \mathrm{~min}$.

7. Incubate the plate for $20 \mathrm{~min}$ at RT.

8. Read the absorption at $630 \mathrm{~nm}$ wavelength in a microwell plate reader.

\section{Data visualization and analysis}

1. Calculate the average absorbance values for the PPase only reaction wells (negative control or background signal). 
NOTE: As a positive control of a SAMHD1 allosteric activator and substrate, dGTP can be included in the plate. In this case, you may use this condition to calculate Z-factor as an indicator of assay quality.

2. Subtract the background value from the corresponding wells in the SAMHD1/PPase reactions.

3. Plot corrected absorbance values for each nucleotide analogue with buffer, GTP, and dGTPaS conditions.

\section{Representative Results}

The protocol outlined in Figure 1 describes the basic workflow for utilizing the enzyme-coupled malachite green assay to probe the interaction of small molecules with the dNTPase SAMHD1 and can be adapted in a number of ways to interrogate different biochemical questions. In the representative results discussed in the below paragraphs, we illustrate examples of using this assay to determine the inhibitory properties of small molecules toward SAMHD1 and to test whether different nucleotide analogues are substrates and/or activators of this enzyme.

The results shown in Figure 2 illustrate several core principles of this assay. The malachite green reagent allows the colorimetric detection of inorganic phosphate through the formation of a phosphomolybdate malachite green complex, and accordingly, this approach can be applied to the study of enzymatic reactions whose product is phosphate. To demonstrate the sensitivity of this method to detect free inorganic phosphate, Figure $2 \mathrm{~A}$ shows the absorbance values obtained with increasing concentrations of $\mathrm{Na}_{3} \mathrm{PO}_{4}$ following a $20 \mathrm{~min}$ incubation with the malachite green reagent. While the signal reaches saturation at $0.25 \mathrm{mM}$
$\mathrm{Na}_{3} \mathrm{PO}_{4}$, the linear detection range of phosphate is visible from 0.004 to $0.03 \mathrm{mM}$ (Figure 2A, right panel), in agreement with other studies that reported a phosphate linear range up to $10-20 \mu \mathrm{M}$ using the malachite green assay ${ }^{38}$.

SAMHD1 is a dNTPase that releases inorganic triphosphate when hydrolyzing a dNTP molecule, and thus in order to generate free inorganic phosphate for detection by malachite green, a coupling enzyme is required. Inorganic pyrophosphatase (PPase) from E. coli has been shown useful for this purpose, both with regard to SAMHD1 ${ }^{7,20}$, but also other nucleotide metabolizing enzymes ${ }^{30,33,35}$. Additionally, SAMHD1 is an active dNTPase when as a homotetramer, and this requires allosteric activation by (d)NTPs, specifically a guanine triphosphate (GTP or dGTP) at AS1 and any dNTP at AS2. Subsequently, the catalytic site becomes accessible for substrate binding and the enzymatic reaction takes place. As dGTP fulfils the requirements for binding to AS1 and AS2, and is a substrate, use of this nucleotide in the inhibition assay greatly simplifies the workflow. Figure 2B illustrates the requirement of the different assay components to achieve measurable SAMHD1 activity indicated by an increase in absorbance at $630 \mathrm{~nm}$. Neither SAMHD1 nor PPase alone are capable of generating inorganic phosphate in the presence of dGTP, consistent with the documented activities of these enzymes. However, in the condition in which all the assay components are present (SAMHD1, PPase, and the dGTP activator/substrate) we observe an increase in signal. The $Z$ factor $^{37}$ of the example shown here (taking no enzymes + dGTP as a negative control and SAMHD1/PPase + dGTP as a positive control) was 0.74 , indicating a robust assay.

One of the potential applications of the enzyme-coupled SAMHD1 activity assay is the identification of inhibitors through high-throughput screening (HTS). Thus, in this report, 
we validate the detection of SAMHD1 inhibition in this assay using a diverse set of compounds already described in the literature. Seamon et al. evaluated the dose-dependent inhibition of canonical nucleosides toward SAMHD1 using a similar assay as shown here, and found that deoxyguanosine (dGuo) was the only canonical nucleoside able to significantly inhibit SAMHD1, with an $\mathrm{IC}_{50}$ value of $488 \mu \mathrm{M}^{20}$. A HTS of FDA-approved drugs performed with the direct b4NPP assay revealed several hits that inhibited SAMHD1 activity at micromolar concentrations, from which lomofungin was the molecule that most potently inhibited SAMHD1 dNTPase activity in vitro, exhibiting an $\mathrm{IC}_{50}$ of $20.1 \mu \mathrm{M}$ when determined in the presence of dGTP as a substrate ${ }^{21}$. Additionally, the four $\alpha, \beta$-imido-dNTP analogs have also been identified as competitive inhibitors of SAMHD1 using the MDCC-PBP sensor and SAMHD1 coupled to Ppx activity, which showed that the inhibitory constants of the dNMPNPP analogs were in the low micromolar / high nanomolar range $^{6,22}$. Thus, to demonstrate that the enzyme-coupled SAMHD1 activity assay can be used to identify SAMHD1 inhibitors, dGuo, lomofungin and 2'-deoxythymidine-5'-[( $\alpha, \beta)$ imido]triphosphate (dTMPNPP), were used to validate the technique. Figure 3A illustrates the dose-response curves obtained for these compounds, showing that increasing concentrations effectively inhibit SAMHD1 activity. The mean IC50 values obtained for these molecules from three independent experiments ( \pm standard deviation) were as follows: $\mathrm{dGuo}=361.9 \pm 72.8 \mu \mathrm{M}$, lomofungin $6.78 \pm 3.9$ $\mu \mathrm{M}$, and dTMPNPP $=2.10 \pm 0.9 \mu \mathrm{M}$. As an example of a negative result, the impact of hydroxyurea (HU) on SAMHD1 activity was also determined. $\mathrm{HU}$ is an inhibitor of ribonucleotide reductase, and, although it limits SAMHD1 araCTPase activity in various AML models, the effects of $\mathrm{HU}$ on SAMHD1 were shown to be indirect and rely on perturbing the allosteric regulation of SAMHD1 ${ }^{18}$. The dose response curve of $\mathrm{HU}$ is shown in Figure $3 \mathbf{B}$, and no changes in SAMHD1 activity were observed with increasing HU doses, demonstrating that $\mathrm{HU}$ does not inhibit SAMHD1 activity in vitro.

Another use of the enzyme coupled SAMHD1 activity assay is to interrogate whether nucleotides and their analogs are substrates and/or allosteric activators of this enzyme, which is illustrated in Figure 4. In this experiment, canonical nucleotides, as well as the active metabolites of several anticancer nucleoside analogs, such as cytarabine (ara-CTP), clofarabine (Cl-F-ara-ATP), and gemcitabine (dF-dCTP), were tested as SAMHD1 substrates and activators. Due to the complex allosteric regulation of SAMHD1, the reaction is performed in the presence of GTP as an AS1 activator or the non-hydrolysable dGTP analog 2'-deoxyguanosine-5'(a-thio)-triphosphate (dGTPaS), which can occupy AS1 and AS2. SAMHD1 activity in the presence of the tested nucleotide analog and GTP indicates that the nucleotide is able to bind to the secondary allosteric site and catalytic site (i.e., AS2 activator and substrate), while SAMHD1 activity with the nucleotide analog and dGTPaS indicates the nucleotide can only occupy the catalytic site (i.e., only a substrate). If the nucleotide is able to bind to both the AS1 and AS2 allosteric sites and to the catalytic site, SAMHD1 will be active in the presence of the nucleotide alone, as shown in the case of dGTP. The results show that all canonical dNTPs are able to bind to the AS2 site and to the catalytic site. In the case of nucleotide analogs, clofarabine triphosphate is an AS2 activator and a substrate, whereas cytarabine triphosphate is only able to occupy the catalytic site. On the other hand, no activity was observed with gemcitabine triphosphate, suggesting that under the conditions tested gemcitabine triphosphate is not able to act as allosteric effector nor substrate. Although this result is consistent 
with previous predictions ${ }^{9}$, later crystallization and kinetic studies ${ }^{10}$ revealed that gemcitabine triphosphate is able to bind the SAMHD1 catalytic pocket, and that it is indeed a substrate of the enzyme. However, in the latter study ${ }^{10}$, the authors show that the hydrolysis rate is considerably lower compared to other reported substrates, such as cytarabine triphosphate, thus explaining why we were not able to observe this with this screening setup.
Altogether, these representative results validate the use of the enzyme coupled SAMHD1 activity assay as a robust technique for the identification and characterization of SAMHD1 inhibitors, allosteric regulators, and substrates. However, similar to all experimental approaches, this method has its caveats, and so orthogonal assays (e.g., using a different assay technology) should be used to further validate findings. 


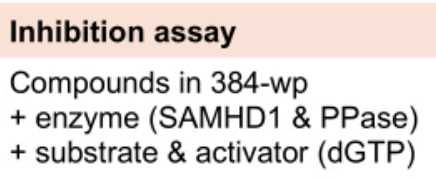

\section{Activator/substrate assay}

Nucleotides in 384-wp

+ AS1/AS2 activator (GTP/dGTPaS)

+ enzyme (SAMHD1 \& PPase)

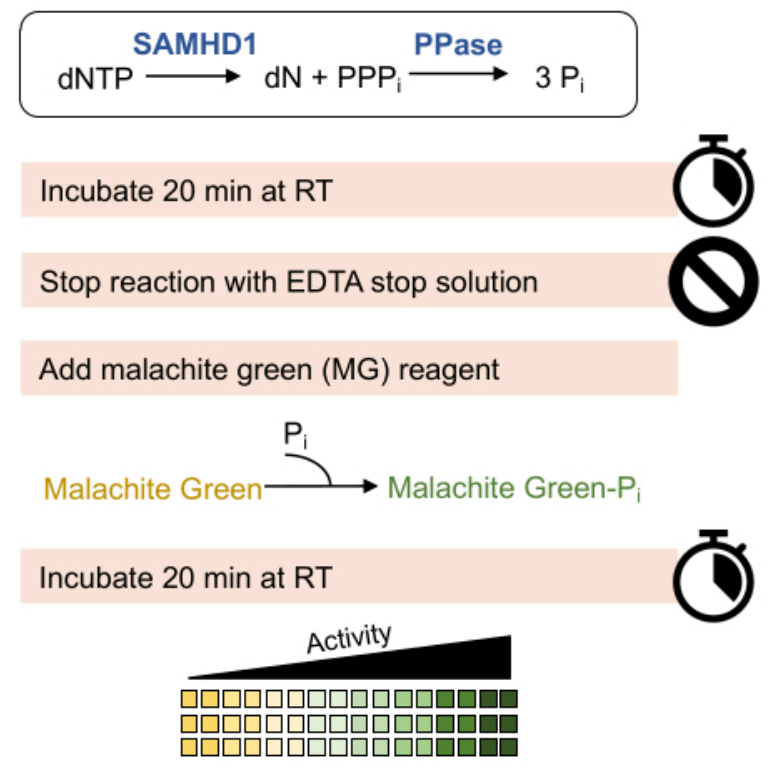

Read plate @ 630 nm and perform analysis
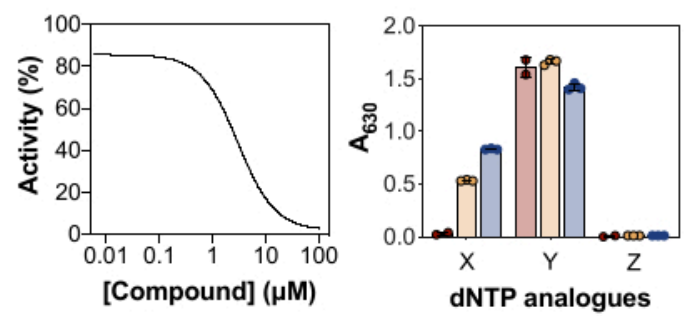

Figure 1: Schematic overview of the protocol described in this article. Please click here to view a larger version of this figure. 
A
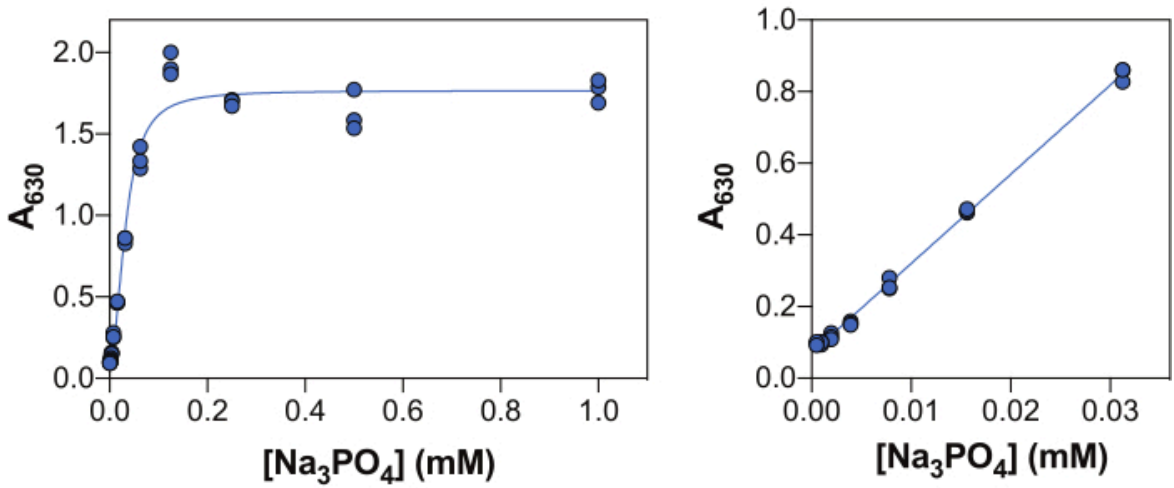

B

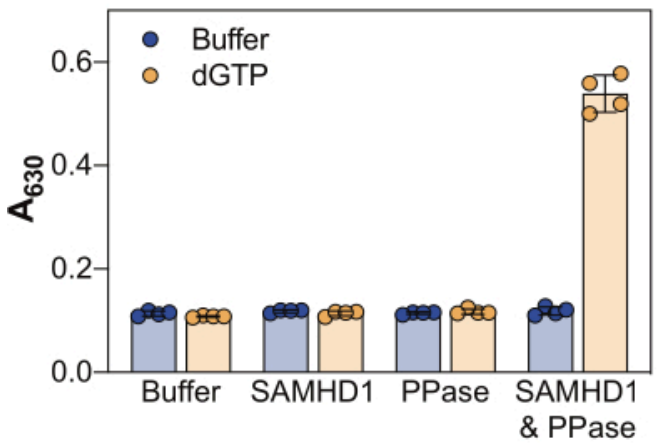

Figure 2: Enzyme-coupled SAMHD1 activity assay. (A) $\mathrm{Na}_{3} \mathrm{PO}_{4}$ standard curve in the malachite green assay. $\mathrm{Na}_{3} \mathrm{PO}_{4}$ serial dilution (2-fold) was prepared from $1 \mathrm{mM}$ to $0.004 \mathrm{mM}$ in triplicate and incubated with malachite green reagent for 20 min. Raw absorbance values over the full range of tested concentrations are shown in the left panel and the linear range in the right panel. Representative of two independent experiments shown. (B) Validation of enzyme-coupled activity assay. SAMHD1 $(0.35 \mu \mathrm{M})$ and/or PPase $(12.5 \mathrm{U} / \mathrm{mL})$ in the presence or absence of activator/substrate dGTP $(25 \mu \mathrm{M})$ were incubated for $20 \mathrm{~min}$ in the enzyme-coupled activity assay. Quadruplets from a representative of two independent experiments shown with raw absorbance values plotted, bars, and error bars indicate mean and SD. Please click here to view a larger version of this figure. 
A

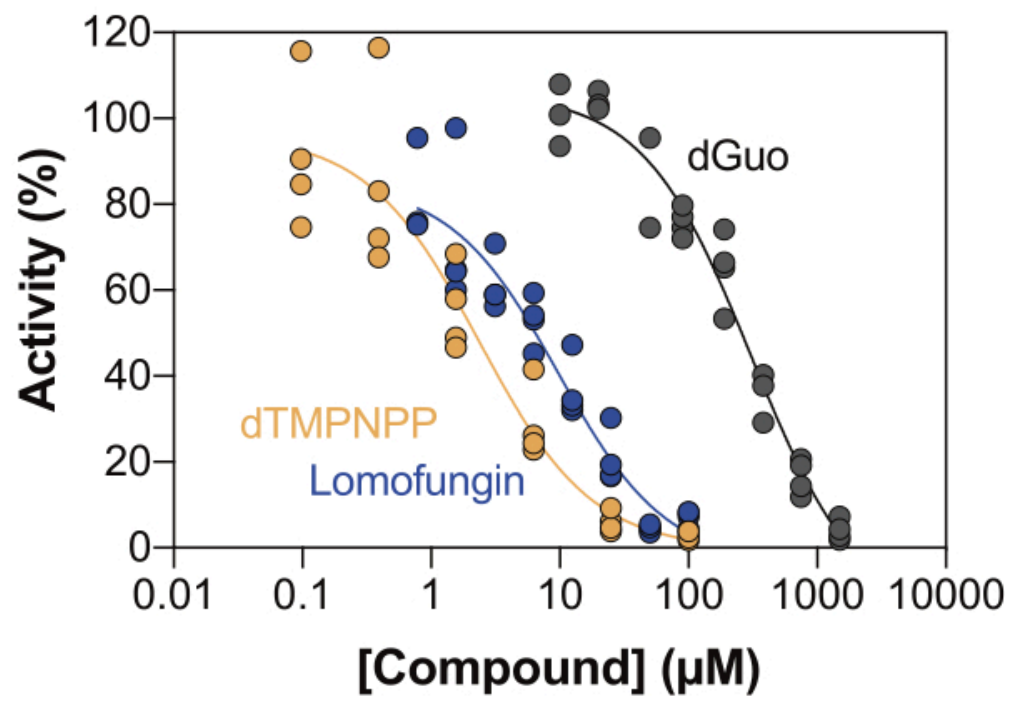

B

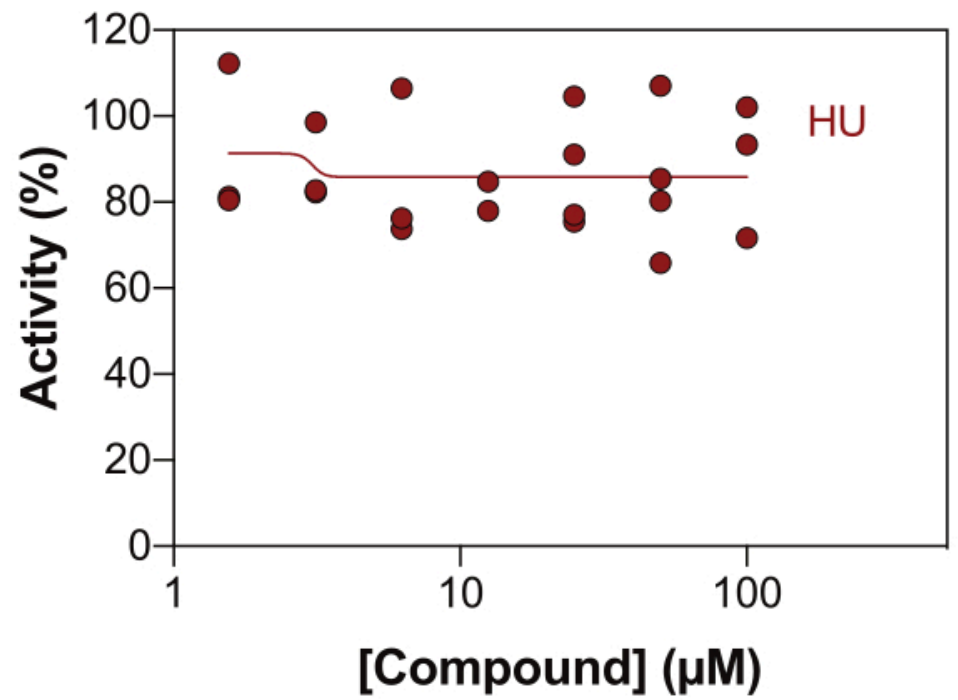

Figure 3: Evaluating compounds for SAMHD1 inhibition in the enzyme-coupled activity assay. Dose response of lomofungin (0.78-100 $\mu \mathrm{M}), 2^{\prime}-$-deoxythymidine-5'-[( $\left.\alpha, \beta\right)$-imido]triphosphate (dTMPNPP, 0.01-100 $\left.\mu \mathrm{M}\right)$ and deoxyguanosine (dGuo, 10-1,500 $\mu \mathrm{M})(\mathbf{A})$ or hydroxyurea $(\mathrm{HU})(0.78-100 \mu \mathrm{M})(\mathbf{B})$ in the enzyme-coupled SAMHD1 activity assay with dGTP $(25 \mu \mathrm{M})$ as activator/substrate. Percentage activity relative to reaction controls from individual replicates plotted (DMSO + SAMHD1/PPase + dGTP $=100 \%$ activity, DMSO + dGTP $=0 \%$ activity) with a representative of three experiments shown. 


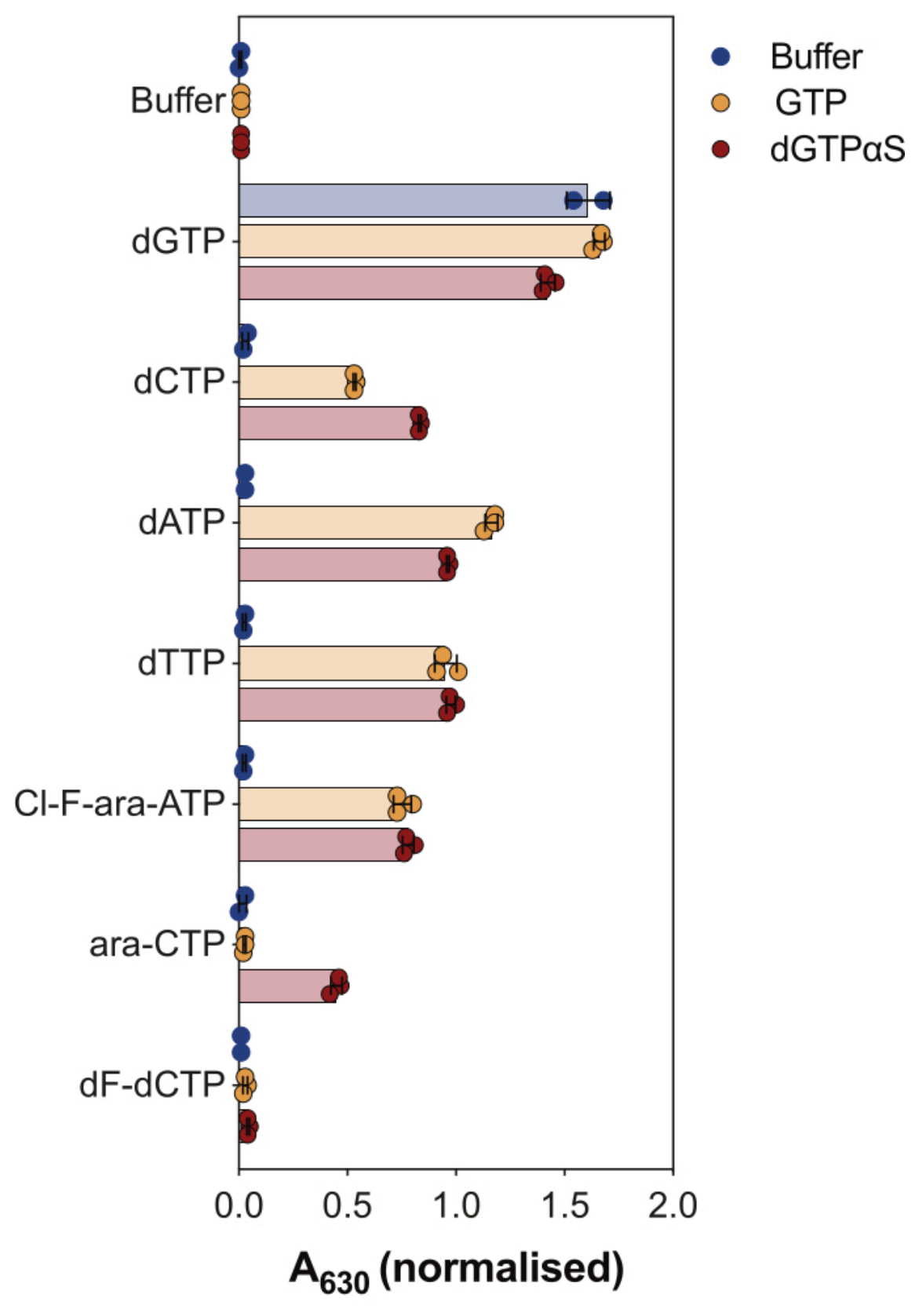

Figure 4: Evaluating nucleotide analogs as SAMHD1 allosteric activators and substrates in the enzyme-coupled activity assay. Canonical nucleotides and selected triphosphate metabolites of anticancer drugs cytarabine (ara-CTP), clofarabine (Cl-F-ara-ATP), and gemcitabine (dF-dCTP), were tested at $200 \mu \mathrm{M}$ in the enzyme-coupled SAMHD1 activity assay in the presence or absence of GTP or non-hydrolysable dGTP analog dGTPaS (12.5 $\mu \mathrm{M})$. Normalized absorbance values from individual experimental replicates plotted, mean and SD are indicated. Representative of two independent experiments shown, adapted from our previous study ${ }^{7}$. Please click here to view a larger version of this figure. 


\begin{tabular}{|c|c|c|c|c|c|c|}
\hline Step & Reagent & $\begin{array}{c}\text { Volume } \\
\text { dispensed }(\mu \mathrm{L})\end{array}$ & $\begin{array}{l}\text { Final reaction } \\
\text { volume }(\mu L)\end{array}$ & $\begin{array}{c}\text { Concentration } \\
\text { dispensed }\end{array}$ & $\begin{array}{l}\text { Fold dilution } \\
\text { in reaction }\end{array}$ & $\begin{array}{c}\text { Final } \\
\text { concentration } \\
\text { in reaction }\end{array}$ \\
\hline 1 & Inhibitor & 5 & \multirow[t]{3}{*}{20} & $0.4 \mathrm{mM}$ & 4 & $0.1 \mathrm{mM}$ \\
\hline 2 & $\begin{array}{c}\text { SAMHD1+PPase } \\
\text { mix }\end{array}$ & 5 & & $\begin{array}{c}1.4 \mu \mathrm{M} \\
\text { SAMHD1, } 50 \\
\text { U/mL PPase }\end{array}$ & 4 & $\begin{array}{c}0.35 \mu \mathrm{M} \\
\text { SAMHD1, } 12.5 \\
\text { U/mL Ppase }\end{array}$ \\
\hline 3 & dGTP & 10 & & $50 \mu \mathrm{M}$ & 2 & $25 \mu \mathrm{M}$ \\
\hline 4 & \multicolumn{6}{|c|}{ Incubation for 20 minutes } \\
\hline 5 & EDTA solution & 20 & 40 & $7.9 \mathrm{mM}$ & 2 & $3.95 \mathrm{mM}$ \\
\hline 6 & MG reagent & 10 & 50 & $\begin{array}{c}2.5 \mathrm{mM} \\
\text { Malachite } \\
\text { green, } 64.4 \\
\text { mM Ammonium } \\
\text { Molybdate, } \\
0.18 \% \\
\text { Tween-20 }\end{array}$ & 5 & $\begin{array}{c}0.5 \mathrm{mM} \\
\text { Malachite } \\
\text { green, } 12.9 \\
\text { mM Ammonium } \\
\text { Molybdate, } \\
0.036 \% \\
\text { Tween-20 }\end{array}$ \\
\hline 7 & \multicolumn{6}{|c|}{ Incubation for 20 minutes } \\
\hline 8 & \multicolumn{6}{|c|}{ Read @630 nm } \\
\hline
\end{tabular}

Table 1: Summary of the final conditions in the enzyme-coupled assay for inhibitors screening. 


\begin{tabular}{|c|c|c|c|c|c|c|}
\hline Step & Reagent & $\begin{array}{c}\text { Volume } \\
\text { dispensed }(\mu L)\end{array}$ & $\begin{array}{c}\text { Final reaction } \\
\text { volume }(\mu L)\end{array}$ & $\begin{array}{c}\text { Concentration } \\
\text { dispensed }\end{array}$ & $\begin{array}{l}\text { Fold dilution } \\
\text { in reaction }\end{array}$ & $\begin{array}{c}\text { Final } \\
\text { concentration } \\
\text { in reaction }\end{array}$ \\
\hline 1 & $\begin{array}{l}\text { Allosteric } \\
\text { regulator }\end{array}$ & 5 & \multirow[t]{3}{*}{20} & $800 \mu \mathrm{M}$ & 4 & $200 \mu \mathrm{M}$ \\
\hline 2 & GTP or dGTPaS & 5 & & $50 \mu \mathrm{M}$ & 4 & $12.5 \mu \mathrm{M}$ \\
\hline 3 & $\begin{array}{c}\text { SAMHD1 } \\
\text { and/or PPase }\end{array}$ & 10 & & $\begin{array}{c}0.7 \mu \mathrm{M} \\
\text { SAMHD1, } 25 \\
\text { U/mL PPase }\end{array}$ & 2 & $\begin{array}{c}0.35 \mu \mathrm{M} \\
\text { SAMHD1, } 12.5 \\
\text { U/mL PPase }\end{array}$ \\
\hline 4 & \multicolumn{6}{|c|}{ Incubation for 20 minutes } \\
\hline 5 & EDTA solution & 20 & 40 & $7.9 \mathrm{mM}$ & 2 & $3.95 \mathrm{mM}$ \\
\hline 6 & MG reagent & 10 & 50 & $\begin{array}{c}2.5 \mathrm{mM} \\
\text { Malachite } \\
\text { green, } 64.4 \\
\text { mM Ammonium } \\
\text { Molybdate, } \\
0.18 \% \\
\text { Tween-20 }\end{array}$ & 5 & $\begin{array}{c}0.5 \mathrm{mM} \\
\text { Malachite } \\
\text { green, } 12.9 \\
\text { mM Ammonium } \\
\text { Molybdate, } \\
0.036 \% \\
\text { Tween-20 }\end{array}$ \\
\hline 7 & \multicolumn{6}{|c|}{ Incubation for 20 minutes } \\
\hline 8 & \multicolumn{6}{|c|}{ Read@630 nm } \\
\hline
\end{tabular}

Table 2: Summary of the final conditions in the enzyme-coupled assay for allosteric regulators screening

\section{Discussion}

The enzyme-coupled activity assay detailed here is a highthroughput-amenable colorimetric assay allowing the indirect measurement of dNTP hydrolysis by SAMHD1. This method exploits the ability of inorganic PPase from E. coli, which when included in excess in the reaction mixture, converts each inorganic triphosphate generated by SAMHD1 into three individual free phosphates that can be quantified using the simple and economical malachite green reagent. We provide this assay in a 384 microwell plate format, which is ideal for screening of compound libraries, and demonstrate the applicability and versatility of this technique in the identification and characterization of SAMHD1 inhibitors, activators, and substrates.

As with all in vitro biochemical screening assays, there are a number of critical steps and important considerations, and many of these are discussed in-depth in the freely available Assay Guidance Manual ${ }^{39}$. Integrity of the purified 
recombinant enzymes, both SAMHD1 and the coupled enzyme inorganic PPase, is extremely important, and should be confirmed prior to establishing the assay. And accordingly, each new purification of these enzymes should be subject to some level of batch testing, as batch-to-batch variabilities could introduce inconsistencies in results. Ideally, use of an orthogonal direct assay, such as HPLC, which allows detection of both the substrate and reaction product, should be used to verify the dNTP triphosphohydrolase activity of the purified recombinant SAMHD1 being used.

Regarding limitations of this assay, the principle one is that it measures the dNTPase activity of SAMHD1 in an indirect manner, exploiting the activity of inorganic PPase, which has a number of implications. It is important to confirm that PPase possesses little to no activity toward nucleotides used in the assay, and likewise, that inhibitory small molecules identified possess no activity toward PPase. Thus, with regard to screening, a counter-screen against PPase can be an important consideration. The presence of PPase in the reaction also makes it critical to use an orthogonal assay to confirm findings. With regard to direct activity assays, a number of them have been reported to date, including $\operatorname{TLC}^{9,20,23}$ and HPLC ${ }^{9,21}$, which accurately detect substrate exhaustion and product formation. Additionally, the b4NPP assay ${ }^{21}$, which is also high-throughput, could be used to test potential inhibitors; however, it is not ideal to test substrates or allosteric activators. Biophysical assays, such as differential scanning fluorimetry (DSF), which we have previously reported with $\mathrm{SAMHD} 1^{18}$, can also be particularly powerful in identifying and characterizing ligands. Another limitation of the assay, specifically as shown in the setup here for identifying substrates and activators, is the use of the non-hydrolysable dGTP analog dGTPaS as an AS1 and AS2 activator. While this allows activation of SAMHD1 with no observable activity in the assay, dGTPaS is a competitive inhibitor of SAMHD1, and thus the use of high concentrations will inactivate the enzyme. As our understanding of SAMHD1 progresses, future studies could utilize molecules that exclusively occupy each site of SAMHD1, thus negating this potential issue.

As we have shown here, this method is versatile and can be used to address a number of biochemical questions. We have described two variations of this assay, one for the identification of allosteric regulators and substrates of SAMHD1, and another for the characterization of inhibitors, but further adaptations can be made. With regard to potential inhibitors, this assay, being microwell plate-based, makes it well suited for downstream mechanism of action studies 39,40 . Similarly, for further characterization of substrates and allosteric regulators, this technique can be used to determine kinetic parameters of catalysis, as we performed for the active metabolite of cytarabine and clofarabine ${ }^{7}$. However, one drawback is that the enzyme-coupled assay reported here is an endpoint assay, and so, although well-suited for screening, a continuous assay would be better suited for some mechanistic studies. Arnold et al. reported a continuous enzyme-coupled assay that utilizes the biosensor MDCC$\mathrm{PBP}^{6}$, which relies on the use of the periplasmic phosphate binding protein (PBP) labeled with coumarin maleimide (MDCC) fluorophore that can bind to a free phosphate group. MDCC-PBP is very sensitive and enables the quantification of very low phosphate concentrations, with the sensor's time of response being in the millisecond to second timescale.

SAMHD1 plays a number of important functions in human health and disease ${ }^{2}$ many of which could be linked to its central role in the maintenance of intracellular dNTP levels ${ }^{1}$. Thus, identification of a high-quality chemical probe toward 
the dNTPase activity of SAMHD1 would be a powerful tool in defining these links, and the enzyme-coupled assay reported here could be readily employed to identify such probes. Furthermore, as nucleoside-based drugs, many of which are modulated by SAMHD1, are a diverse and important group of therapeutic ${ }^{41}$; chemical probes could be further developed into drugs to target SAMHD1 in the clinical setting, with the aim of enhancing the efficacy of these therapies. It is also critical to understand the full extent of the interaction of these nucleoside-based compounds with SAMHD1, a question which can be addressed utilizing this enzymecoupled assay too. Taken together, the enzyme-coupled SAMHD1 activity assay as reported here, is a low-cost, versatile, high-throughput assay that can be used to further our understanding of this important enzyme.

\section{Disclosures}

The authors have nothing to disclose.

\section{Acknowledgments}

We thank Thomas Lundbäck and members of Thomas Helleday's laboratory for advice and support. Part of this work was facilitated by the Protein Science Facility at Karolinska Institutet/SciLifeLab (http://ki.se/psf), and we acknowledge the National Cancer Institute $(\mathrm{NCl})$, Division of Cancer Treatment and Diagnosis (DCTD), and Developmental Therapeutics Program (DTP) (http://dtp.cancer.gov) for providing a compound. Funding was provided by grants awarded to S.G.R. from the Swedish Research Council (2018-02114), the Swedish Cancer Society (19-0056JIA, 20-0879-PJ), the Swedish Childhood Cancer Fund (PR2019-0014), and Karolinska Institutet.
1. Franzolin, E. et al. The deoxynucleotide triphosphohydrolase SAMHD1 is a major regulator of DNA precursor pools in mammalian cells. Proceedings of the National Academy of Sciences of the United States of America. 110 (35), 14272-14277 (2013).

2. Coggins, S. A., Mahboubi, B., Schinazi, R. F., Kim, B. SAMHD1 functions and human diseases. Viruses. 12 (4), $382(2020)$.

3. Goldstone, D. C. et al. HIV-1 restriction factor SAMHD1 is a deoxynucleoside triphosphate triphosphohydrolase. Nature. 480 (7377), 379-382 (2011).

4. Powell, R. D., Holland, P. J., Hollis, T., Perrino, F. W. Aicardi-Goutieres syndrome gene and HIV-1 restriction factor SAMHD1 is a dGTP-regulated deoxynucleotide triphosphohydrolase. The Journal of Biological Chemistry. 286 (51), 43596-43600 (2011).

5. Morris, E. R., Taylor, I. A. The missing link: Allostery and catalysis in the anti-viral protein SAMHD1. Biochemical Society Transactions. 47 (4), 1013-1027 (2019).

6. Arnold, L. H., Kunzelmann, S., Webb, M. R., Taylor, I. A. A continuous enzyme-coupled assay for triphosphohydrolase activity of HIV-1 restriction factor SAMHD1. Antimicrobial Agents and Chemotherapy. 59 (1), 186-192 (2015).

7. Herold, N. et al. Targeting SAMHD1 with the Vpx protein to improve cytarabine therapy for hematological malignancies. Nature Medicine. 23 (2), 256-263 (2017).

8. Schneider, C. et al. SAMHD1 is a biomarker for cytarabine response and a therapeutic target in acute myeloid leukemia. Nature Medicine. 23 (2), 250-255 (2017).

\section{References}


9. Hollenbaugh, J. A. et al. Substrates and inhibitors of SAMHD1. PloS One. 12 (1), e0169052 (2017).

10. Knecht, K. M. et al. The structural basis for cancer drug interactions with the catalytic and allosteric sites of SAMHD1. Proceedings of the National Academy of Sciences of the United States of America. 115 (43), E10022-E10031 (2018).

11. Oellerich, T. et al. Selective inactivation of hypomethylating agents by SAMHD1 provides a rationale for therapeutic stratification in AML. Nature Communications. 10 (1), 3475 (2019).

12. Herold, N. et al. SAMHD1 protects cancer cells from various nucleoside-based antimetabolites. Cell Cycle. 16. (11), 1029-1038 (2017).

13. Rothenburger, T. et al. SAMHD1 is a key regulator of the lineage-specific response of acute lymphoblastic leukaemias to nelarabine. Communications Biology. 3 (1), 324 (2020).

14. Ordonez, P. et al. SAMHD1 enhances nucleosideanalogue efficacy against HIV-1 in myeloid cells. Scientific Reports. 7, 42824 (2017).

15. Castellví, M. et al. Pharmacological modulation of SAMHD1 activity by CDK4/6 inhibitors improves anticancer therapy. Cancers. 12 (3), 713-719 (2020).

16. Rassidakis, G. Z. et al. Low-level expression of SAMHD1 in acute myeloid leukemia (AML) blasts correlates with improved outcome upon consolidation chemotherapy with high-dose cytarabine-based regimens. Blood Cancer Journal. 8 (11), 98 (2018).

17. Rudd, S. G., Schaller, T., Herold, N. SAMHD1 is a barrier to antimetabolite-based cancer therapies. Molecular \& Cellular Oncology. 4 (2), e1287554 (2017).
18. Rudd, S. G. et al. Ribonucleotide reductase inhibitors suppress SAMHD1 ara-CTPase activity enhancing cytarabine efficacy. EMBO Molecular Medicine. 41, e10419 (2020).

19. Seamon, K. J. et al. Small molecule inhibition of SAMHD1 dNTPase by tetramer destabilization. Journal of the American Chemical Society. 136 (28), 9822-9825 (2014).

20. Seamon, K. J., Stivers, J. T. A high-throughput enzymecoupled assay for SAMHD1 dNTPase. Journal of Biomolecular Screening. 20 (6), 801-809 (2015).

21. Mauney, C. H., Perrino, F. W., Hollis, T. Identification of inhibitors of the dNTP triphosphohydrolase SAMHD1 using a novel and direct high-throughput assay. Biochemistry. 57 (47), 6624-6636 (2018).

22. Morris, E. R. et al. Crystal structures of SAMHD1 inhibitor complexes reveal the mechanism of water-mediated dNTP hydrolysis. Nature Communications. 11 (1), 3165 (2020).

23. Hansen, E. C., Seamon, K. J., Cravens, S. L., Stivers, J. T. GTP activator and dNTP substrates of HIV-1 restriction factor SAMHD1 generate a long-lived activated state. Proceedings of the National Academy of Sciences of the United States of America. 111 (18), E1843-E1851 (2014).

24. Baykov, A. A., Evtushenko, O. A., Avaeva, S. M. A malachite green procedure for orthophosphate determination and its use in alkaline phosphatase-based enzyme immunoassay. Analytical Biochemistry. 171 (2), 266-270 (1988).

25. Hyun, M., Bohr, V. A., Ahn, B. Biochemical characterization of the WRN-1 RecQ helicase 
of Caenorhabditis elegans. Biochemistry. 47 (28), 7583-7593 (2008).

26. Lin, H.-H., Huang, C.-Y. Characterization of flavonol inhibition of DnaB helicase: real-time monitoring, structural modeling, and proposed mechanism. Journal of Biomedicine \& Biotechnology. 2012 (4), 735368 (2012).

27. Yang, M., Wang, G. ATPase activity measurement of DNA replicative helicase from Bacillus stearothermophilus by malachite green method. Analytical Biochemistry. 509, 46-49 (2016).

28. Allard, B., Cousineau, I., Spring, K., Stagg, J. Measurement of CD73 enzymatic activity using luminescence-based and colorimetric assays. Methods in Enzymology. 629, 269-289 (2019).

29. Lee, M. et al. Structure-activity relationship of sulfonyl piperazine LpxH inhibitors analyzed by an LpxE-coupled malachite green assay. ACS Infectious Diseases. 5 (4), 641-651 (2019).

30. Carreras-Puigvert, J. et al. A comprehensive structural, biochemical and biological profiling of the human NUDIX hydrolase family. Nature Communications. 8 (1), 1541 (2017).

31. Valerie, N. C. K. et al. NUDT15 hydrolyzes 6-thiodeoxyGTP to mediate the anticancer efficacy of 6thioguanine. Cancer Research. 76 (18), 5501-5511 (2016).

32. Carter, M. et al. Human NUDT22 is a UDP-glucose/ galactose hydrolase exhibiting a unique structural fold. Structure. 26. (2), 295-303.e6 (2018).
33. Gad, H. et al. MTH1 inhibition eradicates cancer by preventing sanitation of the dNTP pool. Nature. 508 (7495), 215-221 (2014).

34. Page, B. D. G. et al. Targeted NUDT5 inhibitors block hormone signaling in breast cancer cells. Nature Communications. 9 (1), 250 (2018).

35. Zhang, S. M. et al. Development of a chemical probe against NUDT15. Nature Chemical Biology. 16 (10), $1120-1128(2020)$.

36. Michel, M. et al. In silico druggability assessment of the NUDIX hydrolase protein family as a workflow for target prioritization. Frontiers in Chemistry. 8, 443 (2020).

37. Zhang, J., Chung, T., Oldenburg, K. A simple statistical parameter for use in evaluation and validation of high throughput screening assays. Journal of Biomolecular Screening. 4 (2), 67-7 (1999).

38. Baykov, A. A., Evtushenko, O. A., Avaeva, S. M. A malachite green procedure for orthophosphate determination and its use in alkaline phosphatase-based enzyme immunoassay. Analytical Biochemistry. 171 (2), 266-270 (1988).

39. Markossian S. et al. Assay guidance manual. Bethesda (MD): Eli Lilly \& Company and the National Center for Advancing Translational Sciences. Available at: https:// www.ncbi.nlm.nih.gov/books/NBK53196/ (2004).

40. Holdgate, G. A., Meek, T. D., Grimley, R. L. Mechanistic enzymology in drug discovery: a fresh perspective. Nature Reviews Drug Discovery. 17 (2), 115-132 (2018).

41. Tsesmetzis, N., Paulin, C. B. J., Rudd, S. G., Herold, $\mathrm{N}$. Nucleobase and nucleoside analogues: resistance and re-sensitisation at the level of pharmacokinetics, 
pharmacodynamics and metabolism. Cancers. 10 (7), 240 (2018).

Copyright $\odot 2021$ JoVE Creative Commons Attribution-NonCommercial-NoDerivs 3.0 Unsorted

jove.com

April $2021 \cdot 170 \cdot \mathrm{e} 62503 \cdot$ Page 20 of 20

License

Page 20 of 20 